\title{
Erratum: General Green's function formalism for layered systems: Wave function approach [Phys. Rev. B 95, 075421 (2017)]
}

\author{
Shu-Hui Zhang, Wen Yang, and Kai Chang \\ (Received 22 August 2017; published 12 September 2017)
}

DOI: 10.1103/PhysRevB.96.119905

There are two notation problems to be corrected. First, in some equations in Sec. III B, the scatterer $C$ is incorrectly denoted by $S$. Second, $a_{L}\left(a_{R}\right)$ denotes the left (right) surface of scatterer $A$ in Sec. III C, but it denotes the thickness of a unit cell in the left lead $L$ (right lead $R$ ) elsewhere. Moreover, in Secs. IV B and IV C, a certain $a_{L}$ (or $a_{R}$ ) is denoted incorrectly by $a$.

The following corrections for the notations are necessary to eliminate these problems:

(1) On the left-hand side of Eqs. (45b) and (45c), the symbol $m_{0} \in S$ in the subscript should be replaced with $m_{0} \in C$. Similarly, on the left-hand side of Eq. (49), the symbol $m \in S$ in the subscript should be replaced with $m \in C$.

(2) At the end of the first sentence of Sec. III C 1: “... the semi-infinite right lead $R$. ., add a new sentence "Notice that $a_{L}\left(a_{R}\right)$ denotes the left (right) surface of the scatterer $A$ throughout Sec. III C, but it denotes the thickness of a unit cell of the left lead $L$ (right lead $R$ ) elsewhere."

(3) At the end of the second sentence of Sec. IV A: “... follows from Eqs. (46)-(48).", add a new sentence "Here $a_{L}\left(a_{R}\right)$ denotes the thickness of a unit cell in the left lead $L(\operatorname{right}$ lead $R)$. This notation is used throughout this paper except for Sec. III C, where $a_{L}\left(a_{R}\right)$ is used to denote the left (right) surface of scatterer $A . "$

(4) In Eqs. (80a)-(80d) and (93)-(96), the inline expression $\sum_{\alpha} e^{i k_{+, \alpha}^{(L)}\left(m_{L}-m_{0}\right) a} \cdots$ between Eqs. (80d) and (81) and the inline expression $\sum_{\alpha} e^{i k_{+, \alpha}^{(L)}\left(m_{L}-m_{0}\right) a} \ldots$ between Eqs. (81) and (82), the symbol $a$ on the exponential should have a subscript $L$ or $R$, i.e.,

$$
\begin{aligned}
\left(m-m_{R}\right) a & \rightarrow\left(m-m_{R}\right) a_{R}, \\
\left(m-m_{L}\right) a & \rightarrow\left(m-m_{L}\right) a_{L}, \\
\left(m_{L}-m_{0}\right) a & \rightarrow\left(m_{L}-m_{0}\right) a_{L}, \\
\left(m_{R}-m_{0}\right) a & \rightarrow\left(m_{R}-m_{0}\right) a_{R} .
\end{aligned}
$$

Altogether there are 18 replacements.

(5) On the right-hand side of Eq. (76), the symbol $q_{p}$ should be replaced with $a_{p}$.

Obviously, these corrections for the notations do not affect any final result or conclusion of the paper. 